\title{
Simulation and Optimization of Cologne's Tram Schedule
}

\author{
Oliver Ullrich ${ }^{1 *}$, Sebastian Franz ${ }^{1}$, Ewald Speckenmeyer ${ }^{1}$, Daniel Lückerath ${ }^{1,2}$ \\ ${ }^{1}$ University of Cologne, Inst. f. Informatics, Pohligstrasse 1, 50969 Cologne, Germany; \\ ${ }^{2}$ Cologne University of Applied Science, Cologne, Germany; *ullrich@ informatik.uni-koeln.de
}

\begin{abstract}
In many tram networks multiple lines share tracks and stations, thus requiring robust schedules which prevent inevitable delays from spreading through the network. Feasible schedules also have to fulfill various planning requirements originating from political and economical reasons. In this article we present a tool set designed to generate schedules optimized for robustness, which also satisfy given sets of planning requirements. These tools allow us to compare time tables with respect to their applicability and evaluate them prior to their implementation in the field.

This paper begins with a description of the tool set focusing on optimization and simulation modules. These software utilities are then employed to generate schedules for our hometown Cologne's tram network, and to subsequently compare them for their applicability
\end{abstract}

\section{Introduction}

In many tram networks, several lines share resources like platforms and tracks. This results in very dense schedules, with vehicles leaving platforms every minute at peak times. In order to prevent inevitable local delays from spreading through the network, a feasible schedule has to be robust.

Many additional planning requirements of real world tram schedules originate from political, economical and feasibility reasons. Thus it is not sufficient to exclusively consider general criteria like robustness or operational costs when generating time tables. Typical requirements include fixed start times at certain stations, e.g. interfaces to national railway systems, core lines to relieve high passenger load, e.g. for lines which traverse city centers, warranted connections at certain stations, and safety distances to be complied with at bidirectional tracks. In this paper we present an introduction to our project to generate and evaluate robust time tables which also satisfy given sets of planning requirements. We describe a tool chain which enables us to generate optimized schedules, compare their feasibility and evaluate them prior to application to real world networks.
This paper continues with a description of the current state of the project, focusing on our approaches on optimization and simulation (Section 1). We then present some experimental results obtained by applying the described software to our hometown Cologne's tram network (Section 2). The paper closes with a short summary of lessons learned and some thoughts on further research (Section 3).

\section{Simulating and Optimizing Tram Schedules}

Our project 'Computer Aided Traffic Scheduling' (CATS) is built around a database complying with the ÖPNV5 data model released by the Association of German Transport Companies (Verband Deutscher Verkehrsunternehmen, see [21]). Visualization, optimization, and simulation modules are connected via operations on the database and through XML configuration files (see Figure 1). Due to its compliance with the ÖPNV5 data model our framework is capable of working on many European tram networks.

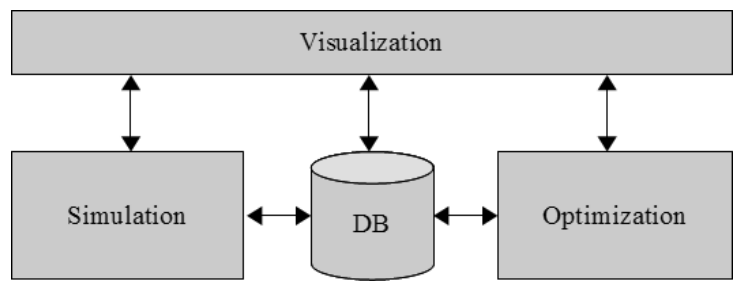

Figure 1. Project modules.

\subsection{Optimization of Tram Schedules}

Various approaches to optimize tram and railway schedules are known (see e.g. $[1,3,4,7,17,18,19]$ ). Most of them aim at one general objective like minimizing vehicle delay (see $[17,19]$ ) or maximizing robustness to restrict the global impact of small, local disturbances (see $[4,7])$. Others apply a combination of objectives, like operational profit and robustness in [3], or combining social opportunity cost and operational cost in [18]. 
Because of the complex nature of the problem, many authors use heuristic approaches like Lagrangian heuristics (see [3]) or simulated annealing (see [18]). Others, like Bampas et al. in [1] introduce exact algorithms for restricted subclasses, like chain and spider networks.

In our project, we combine heuristics and exact methods to generate optimal synchronized time tables for tram networks, targeting maximal robustness and adherence to transport planning requirements at the same time.

We use the scheduled time offset between two consecutive lines departing from a platform as an indicator for robustness. In an assumed tact interval of ten minutes, two lines could be scheduled with equidistant offsets of five minutes, which means that one or both involved vehicles could be late for more than four minutes without consequences for the following tram. Under an extremely unequal split of the available time span into a nine minute offset followed by a one minute offset, the first tram could have a delay of more than eight minutes without consequences to the following vehicle. On the other hand, would the second vehicle be even slightly late, the delay would spread to the followup tram. Since we are assuming typically small delays, we see an equidistant distribution as very robust, the occurrence of very small offsets as not robust.

So, to calculate the robustness of a time table $\lambda$ we examine at each platform $h$ of the network the scheduled time offset $\delta_{f, p r e d(f)}(\lambda, h)$ between any trip $f$ and its predecessor $\operatorname{pred}(f)$, i.e. the time elapsed between the departures of $\operatorname{pred}(f)$ and $f$ at the examined platform.

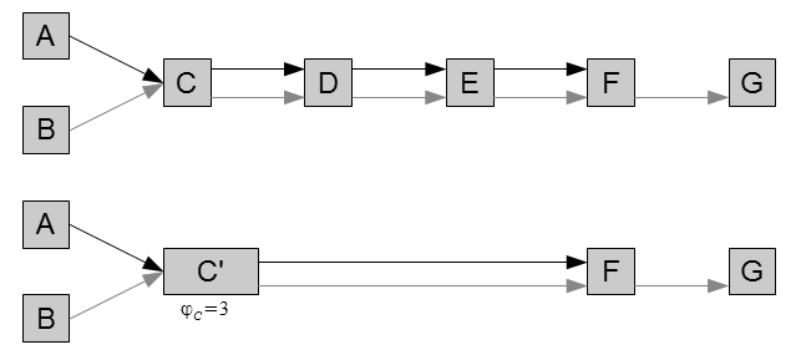

Figure 2. Example of platform reduction.

To reduce complexity we aggregate subsequent similar platforms operated by the same lines to a maximal platform type $h^{\prime}$, weighted by the number of included platforms $\varphi_{h}$ (see Figure 2). The reduced set of platforms is denoted by $H^{\prime}$.
To calculate the robustness $\Phi_{\mathrm{a}}(\lambda)$ of schedule $\lambda$, we add the inverse of $\delta_{f, p r e d(f)}(\lambda, h)$ for all platforms $h \in H^{\prime}$ and all trips, thus applying a penalty for small safety distances. With $F_{h}$ representing all trips that serve platform $h$ under schedule $\lambda$, the resulting function is as follows:

$$
\Phi_{\mathrm{a}}=\sum_{h \in H^{\prime}} \sum_{f \in F_{h}} \frac{1}{\delta_{f, p r e d}(f)(\lambda, h)} * \varphi_{h}
$$

Given is a set $V$ of planning requirements $v \in V$. In order to calculate the compliance with transport planning requirements we introduce $\rho_{v}(\lambda) \in\{1,2,3, \infty\}$, the compliance factor of requirement $v$ under a schedule $\lambda$. A compliance factor 1 means that the requirement is completely satisfied, 2 and 3 denote tolerable compliance, and $\infty$ means that the constraint is not met and the time table candidate $\lambda$ must be rejected. We add the compliance values for all $v \in V$ and get the following:

$$
\Phi_{\mathrm{b}}=\sum_{v \in V} \rho_{v}(\lambda)
$$

Depending on the network under consideration and the number of planning requirements, the two parts of the objective function may not be comparable directly. Thus we define a normalizing factor $\sigma$, which reflects the relationship between the theoretically optimal distance $\delta_{f, p r e d(f)}^{o p t}(h)$ and the best possible compliance factor $\rho_{v}^{\min }$. The theoretically optimal distance $\delta_{f, p r e d(f)}^{o p t}(h)$ of two trips $f$ and $\operatorname{pred}(f)$ on platform $h$ is obtained by dividing the tact interval by the number of serving lines at that platform. The best possible compliance factor $\rho_{v}^{\text {min }}$ of a planning requirement $v$ is the minimal value assigned by the planner, independent of the characteristics of the examined solution candidate. Typically $\rho_{v}^{\min }=1$. We define $\sigma$ as:

$$
\sigma=\frac{\sum_{h \in H^{\prime}} \sum_{f \in F_{h}} \frac{1}{\delta_{f, p r e d(f)}^{o p t}(h)} * \varphi_{h}}{\sum_{v \in V} \rho_{v}^{\min }}
$$

Combining $\Phi(\lambda)_{a}$ and $\Phi(\lambda)_{b}$ yields the overall objective function $\Phi(\lambda)$ (see formula 4), normalized by $\sigma$ and weighted by $\alpha$, the relative weight of the fulfillment of planning requirements.

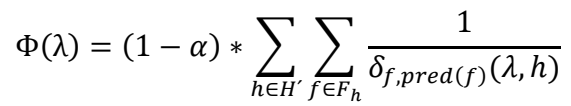

$$
\begin{aligned}
& * \varphi_{h}+\alpha * \sum_{v \in V} \rho_{v}(\lambda) * \sigma
\end{aligned}
$$


We identify seven types of transport planning constraints: Interval constraints, start time constraints, core line constraints, bidirectional track constraints, turning point constraints, warranted connection constraints and follow-up connection constraints. Upon closer inspection it becomes clear that interval and start time constraints are fundamental and all other constraint types can be expressed using these two. E.g. a bidirectional track constraint can be expressed by two interval constraints covering opposing platforms. Subsequently only interval and start time constraints are considered in the remainder of this paper.

A valid solution also has to adhere to some more restrictions. The first restriction requires each start time $\mu_{i}$ to be inside the tact interval, with $t_{\text {interval }}$ being the duration of the interval (see formula 5).

$$
\forall \mu_{i} \in \lambda: \quad 0 \leq \mu_{i}<t_{\text {interval }}
$$

Another restriction requires an offset of at least one minute between two departures $f$ and $\operatorname{pred}(f)$ at each platform $h \in H$ (see formula 6). This means that no platform can be blocked by more than one train at any point of time, the schedule has to be free of collisions.

$$
\forall h \in H: \quad \forall f \in F: \delta_{f, \operatorname{pred}(f)}(\lambda, h)>0
$$

To accelerate the computational process the implemented branch-and-bound solver starts with an initial solution computed by a genetic algorithm. The genetic algorithm encodes a time table as one individual, consisting of the first trip start time of each line, i.e. the offset in minutes from the start of the operational day. All other trips follow by the global tact interval. The application generates a start population using random start time values, testing validity against planning constraints and collisions on network nodes.

To reduce computational complexity we apply simple deterministic tournament selection and two-pointcrossover (as described in [5]). After evaluation of several mutation methods, including random, minimal, and maximum enhancement mutation we choose a minimal random mutation method that only allows start times to be altered by one minute. We utilize a steady state replacement method, also described in [5]. At the end of each run a hill climbing algorithm is applied to the best individual to further improve its fitness.

As described above we use the best individual encountered by the genetic algorithm to provide the branch-and-bound solver with an initial upper bound, thus avoiding a cold start.
Each inner node of the search tree represents a partial solution of the problem. The root of the tree corresponds to a solution in which no line's start time is fixed. With each level of the tree admissible start times for an additional line are set. For a more detailed discussion of the branch-and-bound method, see e.g. [8].

In order to cut branches off the tree as soon as possible, the objective function of the branch-and-bound algorithm is modified. The set of lines $L$ is divided into subset $\hat{L}$ of lines that are already fixed and subset $\tilde{L}$ of lines that are not yet fixed. Accordingly the set of platforms $H$ is divided into $\widehat{H}$ and $\widetilde{H}$. $\widehat{H}$ includes all platforms which are exclusively served by lines already fixed, while platforms in $\widetilde{H}$ are also (or exclusively) served by lines that are not yet fixed. The set of transport planning constraints $V$ is divided into sets $\widehat{V}$ and $\tilde{V}$. Set $\widehat{V}$ includes all constraints which are dependent on already set lines, correspondingly constraints in $\tilde{V}$ are dependent on lines not yet set. The modified objective function $\Phi^{\prime}(\lambda)$ is shown below (formulas 7 to 9).

$$
\begin{aligned}
& \Phi^{\prime}(\lambda)=(1-\alpha) * \Phi^{\prime}{ }_{\mathrm{a}}+\alpha * \Phi^{\prime}{ }_{\mathrm{b}} * \sigma
\end{aligned}
$$

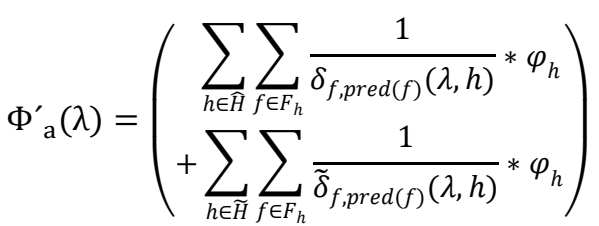

$$
\begin{aligned}
& \Phi^{\prime}{ }_{\mathrm{b}}(\lambda)=\sum_{v \in \widehat{V}} \rho_{v}(\lambda)+\sum_{v \in \widetilde{V}} \rho_{v}^{\min }(\lambda)
\end{aligned}
$$

Here $\tilde{\delta}_{f, p r e d(f)}(\lambda, h)$ represents the theoretically best safety distance value under consideration of lines already fixed. Again, $\rho_{v}^{\min }$ denotes the optimal compliance factor for constraint $v$. These values are applied in order to find a lower bound for solution candidates in the current branch of the search tree. For further implementation details, see [6].

\subsection{Simulation of Tram Schedules}

Most rail-bound traffic simulations are designed for long distance train or railway networks, see e.g. [14, 16]. While those systems feature similarities to tram networks, e.g. passenger exchange or maneuvering capabilities, they differ greatly in important aspects. Tram networks are often mixed, i.e. trams travel on underground tracks as well as on street level, and are thus subject to individual traffic and corresponding traffic regulation strategies. 
Subsequently, tram behavior is a mixture between train and car behavior, e.g. line-of-sight operating / driving. Therefore a simple adaption of railway simulation methodologies is not feasible.

Bearing the similarities with individual traffic in mind Joisten (see [9]) implemented an adapted Nagel/ Schreckenberg model (see [15]) for tram simulation, which suffered from the setbacks of the high aggregation inherent to cellular automatons (see [11]). Therefore Lückemeyer developed an event based simulation model which avoids some of those setbacks as described in [10, 11]. To further eliminate inaccuracies we apply an updated model, is described in detail in the accompanying article 'Modeling time table based tram traffic' ([13]).

Our application is based upon a model-based parallelization framework (described in [20]), which exploits the embedded model's intrinsic parallelism. The mixed tram network is modeled as a directed graph with platforms, tracks and track switches represented by nodes. Connections between nodes are represented as edges. Figure 3 shows part of an example network, which is mapped on the graph depicted in Figure 4, where squares represent platforms, rectangles tracks and triangles track switches. The dark rectangles around platforms indicate that these platforms form a station.

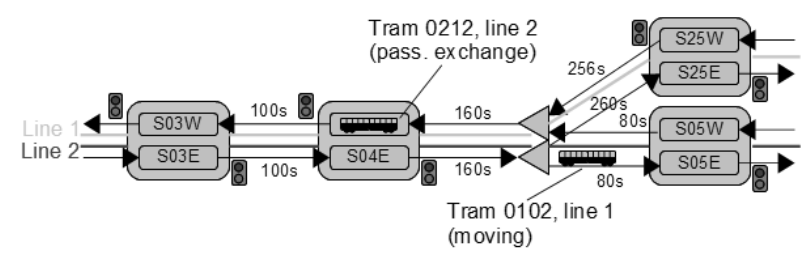

Figure 3. Part of a tram network.

The distributions for the duration of passenger exchange are specific to platform and tram type with the combined duration of opening and closing the vehicle doors as minimum value.

Vehicles encapsulate most of the simulation dynamics, which are based upon the event based simulation approach (as described in [2]). Thus trams change their state at events of certain types, like stopping or accelerating, which happen at discrete points in time. These state changes may trigger a change in the overall system state and generate follow-up events, which are administrated in a priority queue.

Main tram attributes are specified by the type of tram, which holds functions for the maneuvering capabilities, e.g. acceleration and braking.

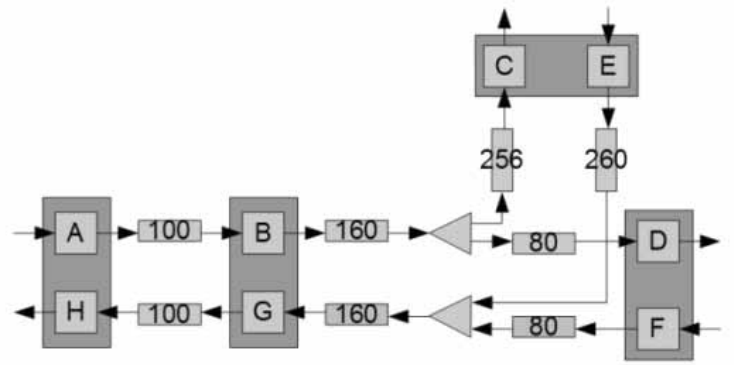

Figure 4. Example graph.

\section{Optimizing Cologne's Tram Network}

We apply the developed software suite to our hometown Cologne's tram network based on the time table data of 2001 (see figure 5). It consists of 528 platforms and 58 track switches connected via 584 tracks, which cover a total length of 407.4 kilometers. 15 lines with 182 line routes are served by 178 vehicles which execute 2,814 trips per operational day.

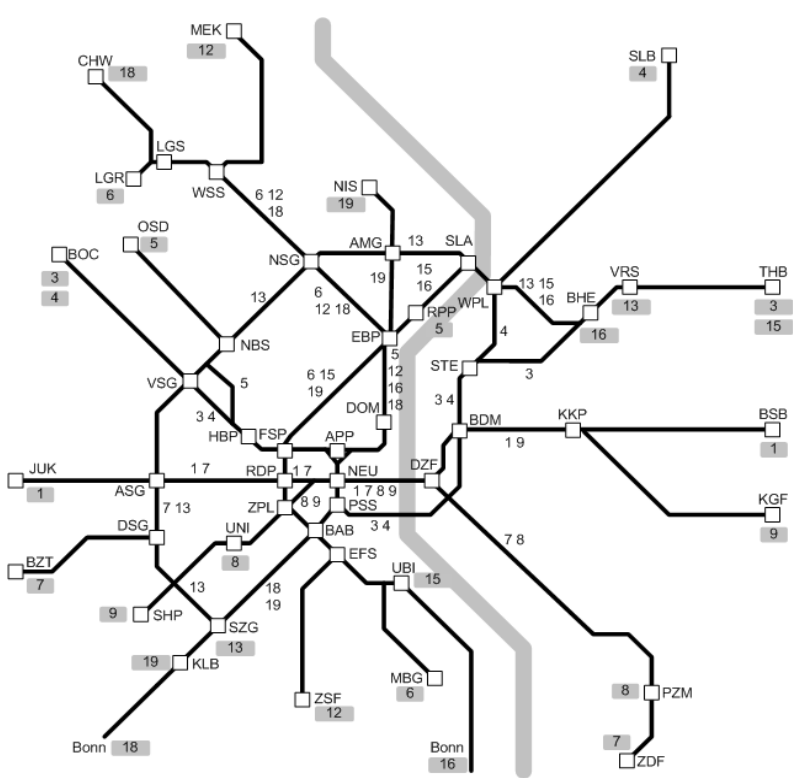

Figure 5. Cologne's tram network in 2001.

For optimization purposes, we only consider the 36 major routes on peak hours. The remaining 146 minor routes are usually trips between the start or end point of a regular trip and depots, or other maintenance trips at the rim of the network. For the following optimization run, we assume a tact interval of ten minutes, and define a set of example constraints, which can be decomposed to two start time constraints and 37 interval constraints. 
These include minimum turn-around times at line ends, an additional core line $1 \mathrm{~A}$ to satisfy high demand for line 1 in Cologne's town center, guaranteed connections between certain lines, and fixed start times at the Bonn national railway hub. A more detailed description of the conducted experiments can be found in [20].

\subsection{Comparing Tram Schedules}

From the genetic algorithm's initial pool of valid solution candidates we randomly pick a schedule A with an objective function value of 214.714 (see Table 1). After a 8.5 hours run, the optimizer yields a pool of 60 best solutions with objective function values of 180.696, from which schedule B (again, see Table 1) is randomly selected.

To begin with a more general view, we pick ten more schedules each out of both solution pools and execute ten simulations runs for each of those 20 schedules. The runs under the initial schedules yield an average delay of departures of 18.9 seconds. Under the best schedules the average delay is 15.4 seconds, which means a reduction of 18.6 percent or 3.5 seconds.

The frequency distribution of occurring delays was also collected (see Figure 6).

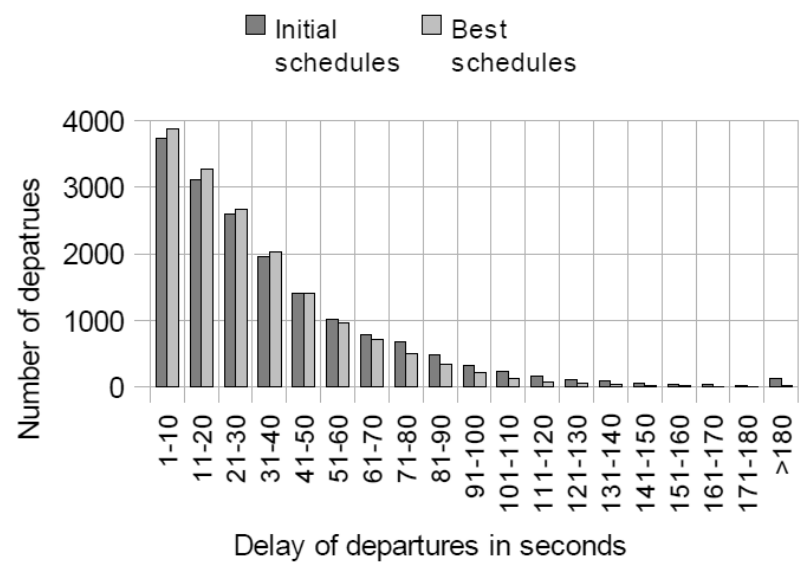

Figure 6. Frequency of all delays.

While the optimal schedules yield more small delays (up to 60 seconds) than the initial schedules, they yield a lower number of bigger delays of more than 60 seconds (see Figure 7): The random schedules result in an average number of 3,095.6 departures with a delay of more than 60 seconds, under the best schedules this number is down by 987.6 departures or 31.9 percent to 2,108.0 departures. The total number of delayed departures is reduced from $16,923.6$ under the random schedules by 602.0 departures or 3.6 percent down to $16,321.6$.
A higher robustness can help to reduce the number of bigger delays by preventing small delays from accumulating over the simulation run. Under optimal schedules with their better distributed time offsets, the inevitable small delays stay small, so their number is higher than under random schedules. On the other hand, bigger delays cannot build up under robust schedules, so their number is smaller than under random schedules.

Furthermore, we examine both schedules A and B by executing 100 simulation runs each and comparing the results. Schedule A yields an average line delay of 16.5 seconds, which gets reduced under schedule B by $16.4 \%$ or 2.7 seconds to 13.8 seconds.

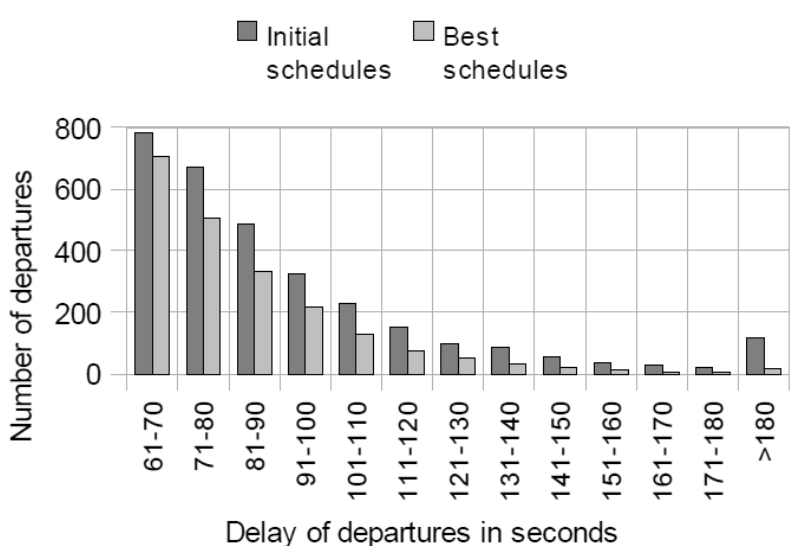

Figure 7. Frequency of delays higher than 60 seconds.

As seen in Figure 8 and Table 2, implementation of schedule B enhances punctuality of most lines at least marginally. Especially the improved punctuality of lines 13 and 18 (32 and 25 percent respectively) show the better coordination between lines under schedule $\mathrm{B}$. Under schedule A, the northbound route of line 13 stands out in coordinating especially bad with line 7 : Although the joining line 7 is scheduled to serve a row of platforms beginning with Dürener Straße/Gürtel (DSG, again see Figure 5) two minutes after line 13, its vehicles often reach the first common track switch ahead of schedule, thus blocking it for the already late trams of line 13. Before entering each of the following common stations, these trams have to wait for line 7 to clear the platforms, thus instantly getting a delay of at least 120 seconds. Only after the end of the shared area at Aachener Straße/Gürtel (ASG), the vehicles can begin to regain part of their lost punctuality.

At the other end of the spectrum, Lines 3, 4, and 5 do not improve on their comparatively low delay, or even yield a slightly higher average delay than under schedule A. 
All three lines are laid out comparatively well in the random schedule A. The westward branch of line 5 is scheduled with an exceptionally high clearance in some areas of the town center, rendering it insusceptible to delays of preceding trains (see Figure 9).

\begin{tabular}{|c|c|c|c|c|}
\hline \multirow{2}{*}{ Line } & \multicolumn{2}{|c|}{ A - Initial schedule } & \multicolumn{2}{c|}{ B - Best schedule } \\
\cline { 2 - 5 } & $\begin{array}{c}\text { For- } \\
\text { ward }\end{array}$ & $\begin{array}{c}\text { Back- } \\
\text { ward }\end{array}$ & $\begin{array}{c}\text { For- } \\
\text { ward }\end{array}$ & $\begin{array}{c}\text { Back- } \\
\text { ward }\end{array}$ \\
\hline $\mathbf{1}$ & 1 & 7 & 7 & 6 \\
\hline $\mathbf{1 A}$ & 6 & 4 & 1 & 0 \\
\hline $\mathbf{3}$ & 3 & 3 & 4 & 0 \\
\hline $\mathbf{4}$ & 7 & 0 & 6 & 7 \\
\hline $\mathbf{5}$ & 5 & 2 & 9 & 6 \\
\hline $\mathbf{6}$ & 1 & 7 & 7 & 6 \\
\hline $\mathbf{7}$ & 0 & 9 & 7 & 0 \\
\hline $\mathbf{8}$ & 6 & 7 & 1 & 7 \\
\hline $\mathbf{9}$ & 5 & 4 & 3 & 7 \\
\hline $\mathbf{1 2}$ & 6 & 7 & 4 & 2 \\
\hline $\mathbf{1 3}$ & 7 & 6 & 0 & 4 \\
\hline $\mathbf{1 5}$ & 4 & 6 & 6 & 9 \\
\hline $\mathbf{1 6}$ & 6 & 0 & 3 & 5 \\
\hline $\mathbf{1 8}$ & 9 & 7 & 6 & 5 \\
\hline $\mathbf{1 9}$ & 9 & 0 & 7 & 9 \\
\hline
\end{tabular}

Table 1. Comparing schedules: Lines.

A closer view on the behavior of line 5 (though under a different schedule) is presented in the accompanying paper 'Modeling time table based tram traffic' (see [13]).

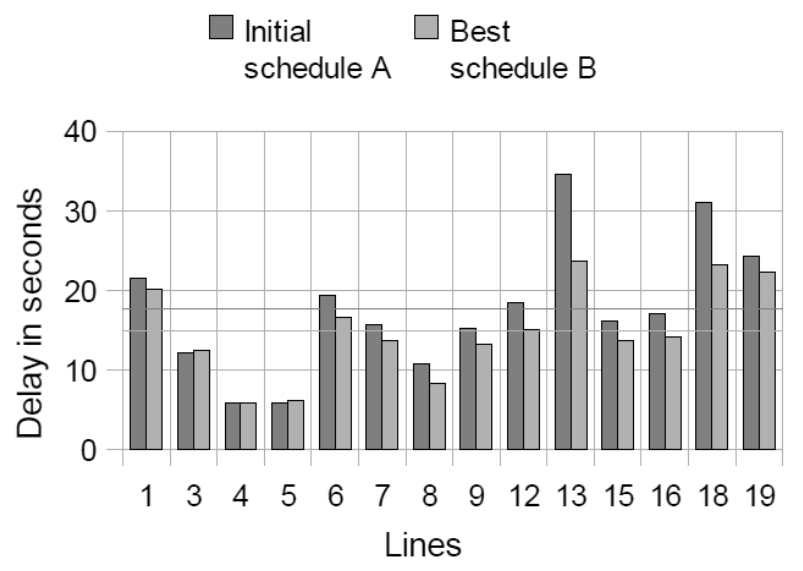

Figure 8. Average delay of lines.
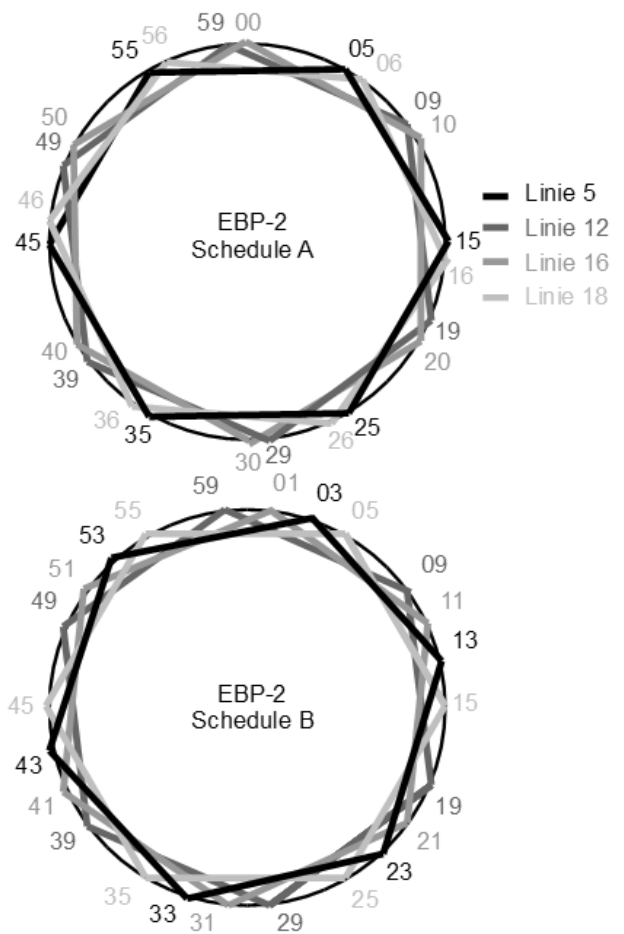

Figure 9. Scheduled departures at platform EPB-2 under schedule $A$ and schedule B.

Simulation data collected at the important hubs Barbarossaplatz (BAB-1 to BAB-4), Ebertplatz (EBP-1 to EBP-4), and Neumarkt (NEU-1 to NEU-4) is presented in Figure 10 and Table 3. Under schedule B, delay was reduced significantly at each of those platforms, on average by 3.7 seconds or 17.6 percent. The increase in punctuality can be explained by the better reliability of the frequenting lines under the optimized schedule.

The high base levels of delay at some platforms (like NEU-2, EBP-4, and BAB-1) is obviously independent of the applied schedule and has therefore to be based on the properties of the surrounding parts of the network.

For instance, the track leading up to NEU-2 has a planned travel time of 120 seconds, including passenger exchange on the platform. It is 880 meters long and because of crossing streets and pedestrian crossings divided by six traffic lights. Because of the applied global phase length of 30 seconds a tram has to wait at each of these lights for 7.5 seconds on average, accumulating to 45 seconds of standing time. Only 75 seconds of scheduled time remain for the actual traversing of the track, including accelerating and braking in front of traffic lights three times on average, and the passenger exchange. This is obviously not enough, resulting in the observed base delay. 


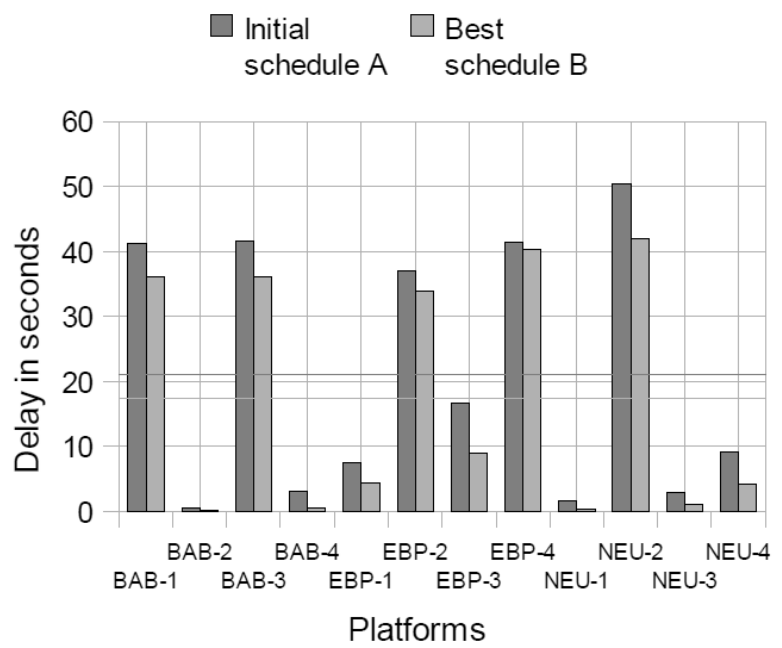

Figure 10. Average delay at platforms.

\begin{tabular}{|c|c|c|c|c|}
\hline \multirow{2}{*}{ Line } & \multicolumn{2}{|c|}{ Average Delay } & \multirow{2}{*}{$\begin{array}{c}\text { Abs. } \\
\text { gain }\end{array}$} & $\begin{array}{c}\text { Rel. } \\
\text { gain }\end{array}$ \\
\cline { 2 - 3 } & $\mathbf{A}$ & $\mathbf{B}$ & 1.4 & 0.06 \\
\hline $\mathbf{1}$ & 21.5 & 20.2 & -0.2 & -0.01 \\
\hline $\mathbf{3}$ & 12.2 & 12.4 & -0.9 & 0.00 \\
\hline $\mathbf{4}$ & 5.9 & 5.9 & 0.0 & -0.06 \\
\hline $\mathbf{5}$ & 5.8 & 6.2 & -0.4 & -14 \\
\hline $\mathbf{6}$ & 19.3 & 16.6 & 2.7 & 0.14 \\
\hline $\mathbf{7}$ & 15.7 & 13.7 & 2.0 & 0.13 \\
\hline $\mathbf{8}$ & 10.8 & 8.3 & 2.5 & 0.23 \\
\hline $\mathbf{9}$ & 15.2 & 13.3 & 1.9 & 0.13 \\
\hline $\mathbf{1 2}$ & 18.4 & 15.0 & 3.4 & 0.19 \\
\hline $\mathbf{1 3}$ & 34.6 & 23.6 & 11.0 & 0.32 \\
\hline $\mathbf{1 5}$ & 16.1 & 13.7 & 2.4 & 0.15 \\
\hline $\mathbf{1 6}$ & 17.0 & 14.1 & 2.9 & 0.17 \\
\hline $\mathbf{1 8}$ & 31.1 & 23.2 & 7.9 & 0.25 \\
\hline $\mathbf{1 9}$ & 24.3 & 22.2 & 2.1 & 0.09 \\
\hline Average & 16.5 & 13.8 & 2.7 & 0.16 \\
\hline
\end{tabular}

Table 2. Comparing schedules: Lines.

A similar situation can be found at other platforms like EBP-4 or BAB-3. The relatively low planned travel times for the up-leading tracks correspond to long tracks with several traffic lights, switches and/or other lines that have to be maneuvered. Thus, a base delay is inevitable.

Other platforms have a lower or almost no base delay: i.e. the platform NEU-1 is preceded by a track with a length of 590 meters with a planned travel time of 180 seconds. The vehicles have to wait at two traffic lights, which leaves enough time to arrive at the platform without delay.

\begin{tabular}{|c|c|c|c|c|}
\hline \multirow{2}{*}{ Platform } & \multicolumn{2}{|c|}{ Average Delay } & \multirow{2}{*}{$\begin{array}{c}\text { Abs. } \\
\text { Gain }\end{array}$} & $\begin{array}{c}\text { Rel. } \\
\text { Gain }\end{array}$ \\
\cline { 2 - 4 } BAB-1 & 41.3 & 36.1 & 5.2 & 0.13 \\
\hline BAB-2 & 0.5 & 0.1 & 0.3 & 0.72 \\
\hline BAB-3 & 41.6 & 36.1 & 5.6 & 0.13 \\
\hline BAB-4 & 3.0 & 0.5 & 2.5 & 0.83 \\
\hline EBP-1 & 7.5 & 4.4 & 3.0 & 0.41 \\
\hline EBP-2 & 36.9 & 33.9 & 3.0 & 0.08 \\
\hline EBP-3 & 16.6 & 8.9 & 7.7 & 0.46 \\
\hline EBP-4 & 41.4 & 40.4 & 1.0 & 0.02 \\
\hline NEU-1 & 1.5 & 0.3 & 1.1 & 0.77 \\
\hline NEU-2 & 50.3 & 41.9 & 8.3 & 0.17 \\
\hline NEU-3 & 2.8 & 1.1 & 1.7 & 0.61 \\
\hline NEU-4 & 9.1 & 4.2 & 5.0 & 0.54 \\
\hline Average & 21.0 & 17.4 & 3.7 & 0.18 \\
\hline & 3.0 & & &
\end{tabular}

Table 3. Comparing schedules: Platforms.

\section{Conclusion and Future Work}

In this paper we presented a tool chain to generate and evaluate tram schedules. The described optimization module is capable of generating robust time tables which fulfill planning requirements of real world projects. We also presented a simulation engine which makes it possible to test real and generated schedules for their applicability and so to further validate them.

We applied the described tool chain to our hometown Cologne's mixed tram network. A random but valid time table A was compared to a resulting best schedule B. As to be expected, the average delay under schedule B is significantly lower than that under schedule A. Most lines and all of the examined core platforms gain punctuality. At least part of the remainig delay can be explained by properties of the underlying network.

In further steps more detailed studies of tram networks and schedules will be carried out, including Cologne's new underground tracks currently under construction, which are designed to relieve the central Neumarkt tunnel. We found it desirable to be able to manually apply small incremental changes to a schedule while getting instant visual assessment of expected consequences. A tool with those capabilities is in the planning stage. Furthermore the optimizer module will be parallelized to further reduce its run time. 
Especially the applied branch-and-bound algorithm's load can be balanced relatively easy, so the application should scale well.

\section{Acknowledgments}

This work was partially supported by Rhein Energie Stiftung Jugend Beruf Wissenschaft under grant number W-10-2-002. We thank the other members of our work group Patrick Kuckertz and Bert Randerath.

An abbreviated version of this paper was previously published in Liu-Henke, X., Buchta, R., Quantmeyer, F. (Ed.): Proceedings of ASIM-Workshop STS/ GMMS 2012. ARGESIM/ASIM Pub. TU Vienna/ Austria, Wolfenbüttel, Feb. 23-24, 2012, 279-289.

\section{References}

[1] E. Bampas, G. Kaouri, M. Lampis, A. Pagourtzis. Periodic Metro Scheduling. In: Proceedings of ATMOS, 2006

[2] J. Banks, J. S. Carson, B.L. Nelson, D. M. Nicol. Discrete-Event System Simulation. Pearson, 2010.

[3] V. Cacchiana, A. Caprara, M. Fischetti. A Lagrangian Heuristic for Robustness, with an Application to Train Timetabling. Transportation Science, to appear.

[4] G. Caimi, M. Fuchsberger, M. Laumanns, K. Schüpbach. Periodic Railway Timetabling with Event Flexibility. In: Networks, 2010, Volume 57, Number 1, pp. 3-18.

[5] J. Dréo, A. Pétrowski, P. Siarry, E. Taillard. Metaheuristics for Hard Optimization. Springer, 2006.

[6] S. Franz. Entwurf und Entwicklung eines mehrstufigen Optimierungsverfahrens für Stadtbahnfahrpläne unter Berücksichtigung verkehrsplanerischen Vorgaben. Diplomarbeit, Univ. Köln, 2011.

[7] Z. Genç. Ein neuer Ansatz zur Fahrplanoptimierung im ÖPNV: Maximierung von zeitlichen Sicherheitabständen. Dissertation, Mathematisch-Naturwissenschaftliche Fakultät, Universität zu Köln, 2003.

[8] T. C. Hu. Combinatorial Algorithms, Addison Wesley, 1982

[9] M. Joisten. Simulation von Fahrplänen für den ÖPNV mittels Zellularautomaten. Diplomarbeit, Univ. Köln, 2002.

[10] G. Lückemeyer. A Traffic Simulation System Increasing the Efficiency of Schedule Design for Public Transport Systems Based on Scarce Data. Dissertation, Shaker Verlag, 2007.
[11] G. Lückemeyer, E. Speckenmeyer. Comparing Applicability of Two Simulation Models in Public Transport Simulation. In: Becker, M., Szczerbicka, H. (Ed.): Proceedings of ASIM 2006, Hannover, 2006.

[12] D. Lückerath. Entwurf und Entwicklung einer Anwendung zur parallelen Simulation von schienengebundenem Öffentlichen Personennahverkehr. Diplomarbeit, Univ. Köln, 2011.

[13] D. Lückerath, O. Ullrich, E. Speckenmeyer. Modeling Time Table based Tram Traffic. SNE Simulation Notes Europe, 22(2), 59-66, ARGESIM Vienna 2012. doi: 10.11128/sne.22.tn.10121

[14] D. Middelkoop, M. Bouwman. SIMONE: Large Scale Train Network Simulations. In: B.A. Peters, J.S. Smith, D.J. Medeiros, M.W. Rohrer (Ed.): Proceedings of the 2011 Winter Simulation Conference, Arlington, 2001.

[15] K. Nagel, M. Schreckenberg. A cellular automaton model for freeway traffic. Journal de Physique I, Volume 2, Issue 12, December 1992, pp. 2221-2229.

[16] A. Nash, D. Huerlimann. Railroad Simulation Using OpenTrack. In: Allan, J., R.J. Hill, C.A. Brebbia, G. Sciutto, S. Sone (Ed.): Computers in Railways IX, WIT Press, Southampton, 2004, pp. 45-54.

[17] A. Schöbel. A Model for the Delay Management Problem based on Mixed-Integer-Programming. In: Proceedings of ATMOS, 2001.

[18] E. Speckenmeyer, N. Li, D. Lückerath, O. Ullrich. Socio-Economic Objectives in Tram Scheduling. Technical Report, Universität zu Köln, to appear.

[19] L. Suhl, T. Mellouli. Managing and preventing delays in railway traffic by simulation and optimization. In: Mathematical Methods on Optimization in Transportation Systems 2001, pp. 3-16.

[20] O. Ullrich. Ein dynamisch-adaptiver Ansatz zur modellbasierten Parallelisierung von Simulationsanwendungen. Mathematisch-Natur-wissenschaftliche Fakultät, Univ. Köln, work in progress.

[21] Verband Deutscher Verkehrsunternehmen e.V. VDVStandardschnittstelle Liniennetz/Fahrplan, VDVSchriften 452, 2008

Submitted: March 2012

Revised: June, 2012

Accepted: August 1, 2012 